Cerebrovasc Dis 2002;14:270

DOI: 10.1159/000065673

\section{Imaging of Stroke Pathology without Predefined Gold Standard}

Rüdiger von Kummer

Department of Neuroradiology, University of Technology, Dresden, Germany

Imaging of patients with acute ischemic stroke is fascinating, because is offers the opportunity to better understand and treat a disabling disease with variable causes and pathophysiology. We appreciated computed tomography (CT) in the early 1970s as the first method demonstrating the diseased brain in vivo. In stroke patients, CT detects hyperattenuating masses and arteries, hypoattenuating brain tissue, and swelling of brain tissue without a change in $\mathrm{x}$-ray attenuation, and we diagnose hemorrhage, intra-arterial thrombus, ischemic brain edema, and tissue swelling due to vasodilatation [1]. Because stroke is fortunately often not a fatal disease, autopsies are rarely done and direct comparison between the image and the stroke pathology is seldom possible. Therefore, we cannot be quite sure that stroke patients without hyperattenuating substrates have really no intracranial hemorrhage. Subtle hemorrhagic tissue transformation of tiny amounts of blood in the cerebrospinal fluid may remain undetected by CT. Stroke patients without hypoattenuating tissue on CT may have no ischemic edema and no irreversible tissue damage, but it is hard to prove. We are missing a gold standard to determine the sensitivity and specificity of brain tissue imaging for certain pathologies. The use of imaging modalities, e.g. the comparison between CT and magnetic resonance imaging (MRI), is of limited value as long as the specificity of findings is undetermined. The recent paper of Jaillard et al. [2] is an example.

Jaillard et al. intended 'to study the pathophysiology of early CT signs'. They compared CT and MRI findings in 16 patients within $29 \mathrm{~h}$ of stroke onset. The CT was obtained $30 \mathrm{~min}$ to $24 \mathrm{~h}$ earlier than MRI (mean 4:48 h) - interestingly, a common finding in papers comparing both modalities [3]. Why is MRI delayed in most studies compared to CT? Based on this comparison with different intervals between both assessments and at different timepoints of the evolving ischemic tissue changes, the authors conclude that early CT signs may show either preinfarction or ischemic penumbra. In particular, they base this conclusion on the observation of patients who had a follow-up T2-weighted MRI (T2WI) that matched the diffusionweighted image (DWI), but was smaller than the early CT findings. The authors do not discuss whether T2WI is really the gold standard for irreversible tissue damage and they do not describe the methods of their follow-up imaging. Moreover, they do not describe which 'CT sign' appeared reversible. It would make sense to find that brain tissue swelling due to compensatory arterial dilatation is reversible if perfusion pressure increases, whereas hypoattenuating tissue represents ischemic edema and irreversible injury [4]. Unfortunately, the images in this paper do not support the authors' conclusions and may even confuse the reader. The legends of figures 2 and 3 are apparently interchanged. The CT scan in figure 2 of patient 2 has a very low window level so that gray and white matter can hardly be distinguished. According to the legend, this CT was obtained at 3:20 h 'after the patient's worsening', according to the figure at 11:20 h, and according to the table at 6:20 h. Despite the low image quality, the hypoattenuating upper lentiform nucleus and central white matter is easily recognized and matches the DWI, $\mathrm{rCBF}$ and $\mathrm{rCBV}$ image at $26 \mathrm{~h}$, and the T2WI at day 5. I wonder why this finding does not appear in figure 1 in the template of patient 2.

The authors still emphasize that the reduction of diffusion is an indicator for irreversible tissue damage. They did not report, however, whether the DWI lesions found in this series of patients matched the lesions on follow-up imaging, e.g. with CT or MRI. They do not discuss why a decrease in ADC triggered by a CBF of $40 \mathrm{ml} /$ $100 \mathrm{~g} \times$ min [5] - far above the penumbra threshold - should mean irreversible injury. Is decreased proton diffusion that can disappear and reapper under experimental conditions [6] really fully explained by 'cytotoxic edema' and does such cytotoxic edema eo ipso mean irreversible damage? Do the authors think that disturbed proton diffusion and hypoattenuating brain tissue represent the same pathology? In my view, it is premature to accept DWI as gold standard for the pathophysiology of ischemic brain tissue. I see ample evidence, however, that hypoattenuating brain tissue after arterial occlusion on CT is a highly specific finding that represents net uptake of water and irreversible injury.

\section{References}

1 Von Kummer R, Bozzao L, Manelfe C: Early CT Diagnosis of Hemispheric Brain Infarction, ed 1. Heidelberg, Springer, 1995.

2 Jaillard A, Hommel M, Baird A, Linfante I, Llinas R, Caplan L, Edelman $\mathrm{R}$, Warach S: Significance of early CT signs in acute stroke. Cerebrovasc Dis 2002;13:47-56.

3 Lansberg M, Albers G, Beaulieu C, Marks M: Comparison of diffusionweighted MRI and CT in acute stroke. Neurology 2000;54:1557-1561.

4 Von Kummer R, Bourquain H, Bastianello S, Bozzao L, Manelfe C, Meier D, Hacke W: Early prediction of irreversible brain damage after ischemic stroke by computed tomography. Radiology 2001;219:95-100.

5 Wang Y, Hu W, Perez-Trepichio A, Ng T, Furlan A, Majors A, Jones S: Brain tissue sodium is a ticking clock telling time after arterial occlusion in rat focal cerebral ischemia. Stroke 2000;31:1386-1392.

6 Li F, Liu K, Silva M, Omae T, Sotak C, Fenstermacher J, Fisher M: Transient and permanent resolution of ischemic lesions on diffusion-weighted imaging after brief periods of focal ischemia in rats. Stroke 2000;31:946954

\section{Rüdiger von Kummer, MD}

Department of Neuroradiology, University of Technology

Fetscherstrasse 74, D-01307 Dresden (Germany)

Tel. +49 351 4582660/5454, Fax +49 3514584370

E-Mail kummer-r@rcs.urz.tu-dresden.de

www.tu-dresden.de/medneurorad

\section{KARGER}

(C) 2002 S. Karger AG, Basel

Fax + 41613061234

E-Mail karger@karger.ch

www. karger.com
Accessible online at

www. karger.com/journals/ced 